\title{
Imbalance of Th17 and T-regulatory cells in peripheral blood and synovial fluid in treatment naïve children with juvenile idiopathic arthritis
}

\author{
JOANNA SZYMAŃSKA-KAEUŻA ${ }^{1}$, BARBARA CEBULA-OBRZUT', PIOTR SMOLEWSKI', \\ JERZY STANCZYK ${ }^{1}$, ELŻBIETA SMOLEWSKA ${ }^{l}$
}

${ }^{1}$ Department of Pediatric Cardiology and Rheumatology, Medical University of Lodz, Poland

${ }^{2}$ Department of Experimental Hematology, Medical University of Lodz, Poland

\begin{abstract}
Objectives: The imbalance between Th17 and T regulatory cells (Tregs) may be a key event in development of autoimmunity. The problem is poorly explored in juvenile idiopathic arthritis (JIA) so far. In this study, peripheral blood $(P B)$ and synovial fluid $(S F)$ Tregs and Th17 cells from were assessed in untreated JIA children.

Material and methods: In 50 children with JIA the PB or SF percentages of Tregs and Th17 cells were assessed by flow cytometry, in comparison with PB Tregs and Th17 cells from 28 healthy controls. Additionally, in both groups the levels of proinfammatory cytokines, such as interleukin (IL)-1 $\beta, I L-6$, $I L-17, I L-21, I L-23$ and tumor necrosis factor $\alpha(T N F-\alpha)$ were assessed using ELISA method.

Results: The proportion of JIA PB Th17 cells was significantly higher than in the controls $(p=0.01)$. Serum levels of IL-1 $\beta, I L-6, I L-17, I L-23$ were also significantly higher in JIA $(p=0.011$, $p=0.007, p=0.008$ and $p=0.023$, respectively). The highest serum IL-6 levels were observed in oligoarthritis JIA $(p=0.031)$. Synovial fluid IL-21 concentration was distinctly higher in polyarticular JIA. Synovial fluid levels of TNF- $\alpha, I L-1 \beta$ and IL-6 were significantly higher than in JIA PB $(p=0.038$, $p=0.013$ and $p<0.001$, respectively). There was a significant correlation between IL-6 and PB Tregs $(p=0.02)$.

Conclusions: The results of this comprehensive analysis indicate a role of Th17 cell activation in the pathogenesis of JIA.
\end{abstract}

Key words: juvenile idiopathic arthritis, Th17 cells, T-regulatory cells, proinflammatory cytokines.

(Centr Eur J Immunol 2014; 39 (1): 71-76)

\section{Introduction}

Juvenile idiopathic arthritis (JIA) is the most common inflammatory arthropathy of the developmental age. Similarly to adult rheumatoid arthritis (RA), a key pathogenetical role may have impaired function of T cells [1]. Additionally, in RA patients activation of cells secreting proinflammatory IL-17 cytokine (Th17), which are suppressed by regulatory T lymphocytes (Tregs), has also been shown [2, 3]. So far, there are only few reports concerning disturbances of Treg and Th17 cell population in children with JIA [4-10].

The lack of an appropriate balance between Th1/Th17 cells and Tregs leads to overcome $\mathrm{T}$ cell tolerance to autoantigens. Activation of acquired immunological response, including interferon $\gamma$ (IFN- $\gamma$ ) and IL-17, provides to acti- vation of innate immunity with contribution of neutrophiles, macrophages or synoviocytes, and stimulates production of several other proinflammatory cytokines such as IL-1, IL- 6 or TNF- $\alpha$, chemokines and inflammatory mediators $[1,4,5]$. Interleukin 21 is essential for maintaining Th17 cells, whereas IL-23 fulfills a role of Th17 phenotype stabilization. In contrast, in the presence of IL- 2 and IFN- $\gamma$ differentiation of Th17 cells is inhibited. Factors inhibiting maturation and proliferation of Th17 cells include also IL-25 and IL-27 [6-8].

Thus, imbalance between Th17 and Tregs may be a key event in development of autoimmunity. The problem is poorly explored in JIA children so far. It was found, that JIA peripheral blood (PB) Tregs are fully functional, whereas in sites of inflammation their supressory function

Correspondence: Prof. Elżbieta Smolewska, Department of Pediatric Cardiology and Rheumatology, Medical University of Lodz, Sporna 36/50, 91-738 Lodz, Poland, e-mail: e.smolewska@wp.pl 
is impaired [9]. Recent data showed susceptibility of effector T cells in JIA SF to suppressive effect of both PB and SF Tregs. This low responsiveness of is probably due to high activity status of T cells in JIA SF [10].

In this study we assessed Th17 and Tregs in different compartments (PB and SF), in association with proinflammatory cytokine levels, for the first time in treatment naïve JIA children.

\section{Material and methods}

\section{Patients and the controls}

Fifty newly diagnosed children with JIA, not treated before with either disease-modyfing drugs or other agents. Fourty girls and 10 boys, aged between 2 and 17 years, entered the study. All patients and their parents were informed about the aim of the study and their informed consents were obtained. The control group consisted of 28 healthy children. The study was approved by the Ethical Committee of Medical University of Lodz, Poland (decision No RNN/416/10/KB).

Majority of the study group comprised children with oligoarthritis type of JIA onset (31 patients, 62\%), whereas 16 children $(32 \%)$ represented polyarticular and only $3(6 \%)$ systemic disease. The type of onset was defined according to ILAR criteria (2001) [11]. Activity of JIA was established based on the JADAS-27 scale criteria [12]. Low, intermediate and high stages of the disease activity have been distinquished accordingly. In the study group $28(53 \%)$ children had a low, $9(18 \%)$ a medium and 13 $(26 \%)$ a high the disease activity (Table 1$)$. All the examined patients children had a normal renal function.

\section{Patient samples}

Peripheral blood samples were obtained during routine laboratory tests. Additionally, in JIA childrens with articular effusion SF was obtained during diagnostic punction. In these samples $T$ cell subpopulations and concentrations of proinflammatory cytokines were investigated and results were compared with respective $\mathrm{PB}$ data.

\section{Assessment of Th17 cell and Tregs}

Subpopulations of Th17 cells and Tregs were assessed by flow cytometry, using FACScanto II cytometer (Becton-Dickinson; San Jose, CA, USA). For IL-17 cells detection $20 \mu \mathrm{l}$ of anti-CD25 (FITC-conjugated; Becton Dickinson, San Jose, CA, USA) and anti-CD4 (APC-conjugated; Becton Dickinson, San Jose, CA, USA) monoclonal antibodies were added to $50 \mu \mathrm{l}$ of the whole blood. Then, samples were incubated for $20 \mathrm{~min}$ at room temperature, in the dark. After incubation "lyze-no wash" procedure was performed. Then, cells were washed in PBS and centrifuged (5 $\mathrm{min} / 250 \mathrm{~g}$ ) and incubated

Table 1. Clinical and laboratory characteristics of the juvenile idiopathic arthritis (JIA) group

\begin{tabular}{|c|c|}
\hline Clinical parameters & $N(\%)$ \\
\hline total number of JIA patients & $50(100)$ \\
\hline girls & $40(80)$ \\
\hline boys & $10(20)$ \\
\hline oligoarthritis & $31(62)$ \\
\hline polyarticular & $16(32)$ \\
\hline systemic & $3(6)$ \\
\hline low & $28(56)$ \\
\hline JIA activity & $9(18)$ \\
\hline high & $13(26)$ \\
\hline laboratory parameters & Mean values $\pm \mathrm{SD}$ or $N(\%)$ of positivity \\
\hline white blood cell (WBC) count & $11.2 \mathrm{G} / 1$ \\
\hline platelets (PLT) count & $385 \mathrm{G} / 1$ \\
\hline C-reactive protein (CRP) & $8.5 \mathrm{mg} / \mathrm{l}$ \\
\hline rheumatoid factor $(\mathrm{RF})$ - positive & $7(14)$ \\
\hline anti-nuclear antibodies (ANA) - positive & $16(32)$ \\
\hline anti-CCP antibodies - positive & $4(8)$ \\
\hline
\end{tabular}


for 20 min with FIX/Perm buffer (at room temperature, in the dark). Afterward, cells were washed in PBS and centrifuged ( $5 \mathrm{~min} / 250 \mathrm{~g}$ ), then re-suspended in FIX/-Perm buffer and incubated for $15 \mathrm{~min}$ (at room temperature, in the dark). After subsequent washing up and re-suspension in PBS, 20 $\mu \mathrm{l}$ of anti-IL-17A PE-conjugated monoclonal antibody (BD Bioscience, San Jose, CA, USA) was added and cells were incubated for $30 \mathrm{~min}$ (at room temperature, in the dark). After that, cells were washed twice, re-suspended with PBS, and the fluorescence was measured using flow cytometry. Cells with CD4+/CD25-/IL-17+ immunophenotype were recognized as IL-17 lymphocytes. The percentage of T17 cells among population of mononuclear cells, gated based on side scatter (SSC) and forward scatter distribution (FSC), was calculated.

Tregs were assessed using Human Treg Flow Kit (FOXP3 AlexaFluoor 488/CD4 PE-Cy5/CD25PE) (BioLegend, San Diego, CA, USA). The peripheral whole blood sample $(100 \mu \mathrm{l})$ was treated according to standard 'lyze-no wash' procedure. Then, they were incubated with $20 \mu \mathrm{l}$ of anti-CD4/anti-CD25 monoclonal antibody cocktail (for $20 \mathrm{~min}$, at room temperature, in the dark). Afterward, cells were washed in the BioLegend cell staining buffer and centrifuged ( $5 \mathrm{~min} / 250 \mathrm{~g})$. Cells was then washed, re-suspended and incubated for 20 min with BioLegend FIX/Perm buffer (at room temperature, in the dark). This procedure was repeated twice. After spinning down the cells and discarding the supernatant, the sample was re-suspended again in FOX3 FIX/Perm. Then, $5 \mu$ of Alexa Fluor 488 anti-human FOXP3 antibody was added and incubated for $30 \mathrm{~min}$ (at room temperature, in the dark). After that, cells were washed twice, re-suspended with cell staining buffer, and the fluorescence was measured using flow cytometry. Cells expressing CD4+/CD25 $5^{\text {high }}+/$ FOXP3+ were determined as Tregs. The percentage of Tregs among mononuclear cells, which were gated based on SSC and FSC distribution, was calculated.

\section{Cytokine and other parameter assessment}

Additionally, serum and SF concentrations of IL-1 $\beta$, IL-6, IL-17, IL-21, IL-23, and TNF- $\alpha$ in both JIA children and the controls were evaluated by ELISA method (R\&D; Minneapolis, MN, USA and BioVendor; Brno, Czech Republic, respectively).

Finally, in all JIA children particular laboratory parameters were measured, such as blood morphology, including white blood cell (WBC) and platelet (PLT) counts, erythrocyte sedimentation ratio (ESR), serum C-reactive protein (CRP) levels, rheumatoid factor (RF), antinuclear antibodies (ANA), anti-cyclic citrullinated peptide (anti-CCP) antibodies (Table 1), all by routine methods.

\section{Statistics}

The data obtained was analyzed by the STATISTICA 10.0 software. Mean values and standard deviations (SD), as well as medians and range values were estimated. Normality of data distribution was tested by the $\chi^{2}$ test, than parametric non-parametric Mann-Whitney $U$ tests was implemented. Consequently, correlations between analyzed parameters were done by the Spearman rang test. The $p$ values $<0.05$ was considered as a statistically significant.

\section{Results}

\section{Th17 cell and Tregs in peripheral blood of juvenile idiopathic arthritis children}

The proportion of JIA PB Th17 cells was significantly higher than in healthy children $(1.7 \pm 2.3 \%$ vs. $0.5 \pm 0.8 \%$; $p=0.01)$. However, there were not statistically significant differences in the rates of PB Tregs between JIA children and healthy controls $(21.9 \pm 14.5 \%$ vs. $19.8 \pm 14.3 \%$; $p>$ 0.05 (Fig. 1).

The percentage of PB Th17 did not significantly differ between particular types of JIA onset (oligoarthritis vs. polyarticular vs. systemic: $1.8 \pm 2.4 \%, 1.4 \pm 2.2 \%$ and 1.5 $\pm 1.6 \%$, respectively; $p>0.05$ ). There was significantly higher rate of Tregs in polyarticular $(25.8 \pm 18.17 \%)$ than in systemic $(9.9 \pm 6.7 \%)$ type of onset $(p=0.01)$. The mean Tregs percentage in oligoarthritis was $17.7 \pm 11.5 \%$ (vs. polyarticular or systemic JIA $-p>0.05$ ). However, small number of systemic JIA patients assessed in our study urge a caution in interpretation of this part of statistical data.

There were no relationships between PB Th17 and Treg cell frequency and JIA activity. The mean ratios for low vs. intermediate vs. high activity were $1.8 \pm 1.9 \%$ vs. $2.4 \pm 4.1 \%$ vs. $1.0 \pm 1.0 \%$ for Th17 cells $(p>0.05)$ and

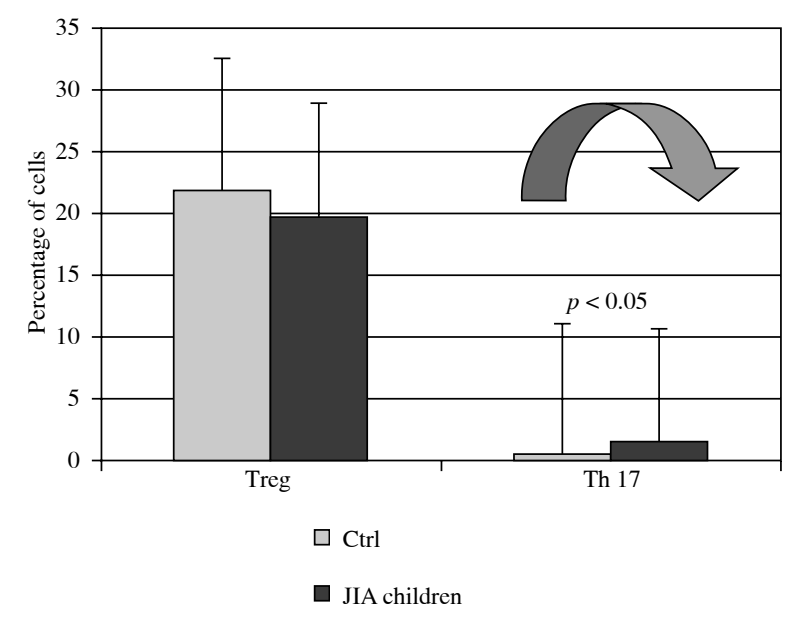

Fig. 1. Differences between interleukin IL-17 secreting (Th17) cells and T-regulatory cells (Tregs) in peripheral blood (PB) from juvenile idiopathic arthritis (JIA) patients and $\mathrm{PB}$ of healthy children $(\mathrm{Ctrl})$ 
$19.9 \pm 13.2 \%$ vs. $19.6 \pm 10.6 \%$ vs. $19.8 \pm 19.2 \%$ for Tregs $(p>0.05)$, respectively.

\section{Th17 cell and Tregs in synovial fluid of juvenile idiopathic arthritis children}

Synovial fluid samples were obtained from 18 JIA patients (83\% with oligoarthritis and $17 \%$ with polyarthritis). The percentages of Th17 cells in PB and SF were comparable $(3.1 \pm 1.8 \%$ vs. $1.7 \pm 1.1 \% ; p>0.05)$, whereas the mean rates of PB Tregs were distinctly higher than in SF $(21.1 \pm 13.5 \%$ vs. $8.3 \pm 10.5 \% ; p=0.035)$.

The percentage of SF Th17 cells was also higher in oligoarthritis children, however, there was not statistically significant difference in comparison to polyarthritis (1.7 $\pm 1.8 \%$ vs. $0.8 \pm 0.9 \%$, respectively; $p>0.05$ ). In oligoarthritis JIA the rate of Tregs in SF was significantly higher than in polyarticular type of onset $(16.0 \pm 11.1 \%$ vs. 2.1 $\pm 0.7 \%$; $p=0.003$ ).

There was no significant correlation between SF Th17 cells frequency and JIA activity. Synovial fluid Treg ratios were distinctly lower in high JIA activity $(6.2 \pm 2.1 \%)$ than in children with low $(15.9 \pm 17.2 \%)$ or medium $(15.8$ $\pm 12.3 \%)$ activity of the disease $(p<0.05)$.

\section{Proinflammatory cytokines: correlation with the disease parameters, Th17 and Treg cells}

Serum levels of IL-1 $\beta$, IL-6, IL-17 and IL-23 were found significantly higher in JIA than in controls $(p=$ $0.011, p=0.007, p=0.008$ and $p=0.023$, respectively) (Table 2).

The highest serum levels of IL-6 were observed in JIA oligoarthritis ( $p=0.031$ ). In turn, SF IL-21 concentration was distinctly higher in polyarticular subtype of the disease. Significantly higher levels of TNF- $\alpha$, IL-1 $\beta$ and IL-6 in JIA SF, as compared to JIA PB were observed ( $p=$ $0.038, p=0.013$ and $p<0.001$, respectively). There was significant correlation between IL-6 serum concentrations and JIA PB Tregs $(\mathrm{R}=0.31, p=0.020)$.

Levels of TNF- $\alpha$, IL- $1 \beta$ and IL- 6 were significantly higher in SF than in JIA serum ( $p=0.038, p=0.013$ and $p<0.001$, respectively). In contrast, concentrations of IL-21 and IL-23 were higher in JIA serum than in SF $(p>0.05)$ (Table 2).

\section{Th17 cells and Tregs and indicators of inflammatory process in juvenile idiopathic arthritis}

In JIA PB, but not in SF, a significant relationship between Tregs and platelet levels was observed $(p=0.028)$. There was no significant correlation between Tregs and other indicators of inflammatory process (WBC count, ESR, CRP levels). Similarly, no correlations were found between Th17 cells and indicators of inflammation.

\section{Discussion}

This is a the first study performed on a big group of previously treatment nadve JIA children, showing correlations between Th17 cells, Tregs or proinflammatory cytokines in PB and SF. Suppressor function of Tregs may be impaired in rheumatoid process, but the data are not fully clear. de Kleer et al. [13] reported decrease percentage of PB Tregs in JIA children. Similar results showed Stelmaszczyk-Emmel et al. [14] in 12 patients with oligoarthritis and polyarticular type of onset. In contrast, Han et al. [15] reported increase in Treg levels in RA patients. In this study, we did not find statistically significant differences in the proportion of PB Tregs in children with JIA compared with the healthy controls. In contrast, PB Th17 cells ratios in JIA patients were significantly higher than in healthy children, what was in concordance with results of Omoyinmi et al. [16].

Table 2. Serum and synovial fluid (SF) concentrations of proinflammatory cytokines in children with juvenile idiopathic arthritis (JIA) and healthy controls (Ctrl)

\begin{tabular}{|c|c|c|c|c|c|}
\hline \multirow[b]{2}{*}{$\begin{array}{l}\text { Cytokines } \\
\text { (pg/ml) }\end{array}$} & \multicolumn{3}{|c|}{ Serum } & \multicolumn{2}{|c|}{ SF } \\
\hline & $\begin{array}{c}\text { JIA } \\
n=\mathbf{5 0} \\
\text { mean } \pm \text { SD }\end{array}$ & $\begin{array}{c}\text { Ctrl } \\
n=29 \\
\text { mean } \pm \text { SD }\end{array}$ & $\begin{array}{c}\text { JIA vs. Ctrl } \\
p\end{array}$ & $\begin{array}{c}n=18 \\
\text { mean } \pm \text { SD }\end{array}$ & $\begin{array}{c}\text { Serum vs. SF } \\
p\end{array}$ \\
\hline TNF- $\alpha$ & $7.0 \pm 3.2$ & $5.8 \pm 5.9$ & $>0.05$ & $13.5 \pm 14.5$ & 0.038 \\
\hline IL-1 $\beta$ & $2.6 \pm 1.7$ & $1.9 \pm 0.5$ & 0.011 & $11.6 \pm 26.0$ & 0.013 \\
\hline IL-6 & $62.6 \pm 98.2$ & $11.8 \pm 4.9$ & 0.007 & $2962.2 \pm 1273.3$ & $<0.001$ \\
\hline IL-17 & $1.4 \pm 1.2$ & $0.6 \pm 0.4$ & 0.008 & $1.95 \pm 1.0$ & $>0.05$ \\
\hline IL-21 & $656.5 \pm 2157.1$ & $152.7 \pm 126.5$ & $>0.05$ & $206.08 \pm 253.9$ & $>0.05$ \\
\hline IL-23 & $93.5 \pm 139.6$ & $23.5 \pm 43.2$ & 0.023 & $62.74 \pm 97.1$ & $>0.05$ \\
\hline
\end{tabular}


Frequency of Tregs and Th17 cells in PB was higher than in SF. It is possible, that in JIA Treg activity is damped, thus their suppressory effect become to be weaker. In turn, they reach the site of inflammation with delay, what further disturb the immune balance and, in consequence, leads to development of autoimmune arthritis. Proinflammatory cytokines are releasing in the inflammed joints what further suppress function of Tregs, and their convertion to Th17 [17].

In contrast to our data, Nistala et al. [18] showed enhanced rate of SF Th17 in active JIA. However, in their study group were patients already treated with non-steroid inflammatory drugs, steroids or disease-modifying antirheumatic drugs (DMARDs), what can influence the results. Prado et al. [17] reported, that SLE patients treated with steroids have increased Th17/Th1 proportion, compared to untreated patients or healthy persons.

We found a tendency to higher percentage of PB Tregs in polyarthritis compared to oligoarthritis and systemic disease, while a higher frequence of Th17 cells in oligoarthritis. However, the differences were not statistically significant. Slightly higher SF Th17 cell percentages was also found in oligoarthritis. There was no relationship between the percentage of both JIA PB and Treg or Th17 cells and JIA activity.

In order to search for association between Th17/Treg cell imbalance previously showed in autoimmune disorders [19] and indicated impairment of the cytokine network, we analyzed concentrations of several proinflammatory cytokines in both serum and SF. In treatment nadve JIA children serum levels of IL-1 $\beta$, IL-6, IL-17, IL-23 were significantly higher than in the control group. Similarly, Prahalad et al. [20] reported significantly higher serum concentrations of IL- $1 \beta$, IL- 6 , and TNF- $\alpha$, in JIA patients examined under treatment with disease-modyfing drugs, as compared to healthy children. Moreover, our study showed that in JIA SF the levels of TNF, IL- $1 \beta$ and IL- 6 were significantly higher compared to JIA PB. In contrast, IL-6 and IL-17 concentrations in SF were lower than in JIA serum.

In the group of children with JIA cytokine levels in serum and SF were also compared depending on the type of the disease onset. Significantly higher levels of IL- 6 in the sera of patients with oligoarthritis JIA onset were observed. Concentrations of TNF- $\alpha$ and IL- $1 \beta$ levels in serum were comparable in all subgroups of patients, and the average values of IL-21 were higher in children with polyarthritis. The concentration of IL-21 in SF was significantly higher in children with polyarticular subtype than those with oligoarticular JIA. These data may confirm that classical proinflammatory cytokine (IL- 6 , IL- $1 \beta$ and TNF- $\alpha$ ) play an important role in initiation and maintaining of the local inflammation, through differentiation of Th17 cells or suppression of Tregs function. Recently, Mahendra et al. [21] reported increased IL-6 and IL-17 levels in SF of children with enthesitis-related arthritis (ERA) JIA subtype, what confirms our data obtained in polyarticular and oligoarticular type JIA onset. Also Agarwal et al. [22] reported elevated levels of IL-17 in SF of patients with both ERA and polyarticular type of JIA onset. On the other hand, de Jager et al. [23] observed elevated IL-6 levels in both JIA PB and SF, although in children with active disease SF IL-6 was significantly higher than in serum.

Moreover, in our study we examined correlation between the serum concentrations of proinflammatory cytokines and the percentages of Th17 and Tregs in PB and SF. We found a significant correlation in PB between IL-6 concentration and Treg percentage.

In addition, in children with JIA the correlation of the percentage regulatory and Th17 cells in PB and SF with basic laboratory indicators of inflammation such as WBC, PLT, ESR, CRP was analysed. In PB, a significant relationship between the percentage of Tregs and platelets levels was observed. However, in the synovial fluid no significant correlation was shown.

In summary, in JIA children the ratio of PB Th17 cells is significantly higher than in healthy subjects. Juvenile idiopathic arthritis SF concentrations of TNF- $\alpha$, IL- $1 \beta$, IL- 6 and IL-17 are higher then in serum of JIA children, what can be responsible for the persistent inflammation in involved joints. We found correlation between PB Treg ratios and serum IL-6 levels, what may confirm the role of that cytokine on Th17 phenotype differentiation. These disturbances of Tregs, Th17 cells and inflammatory cytokines in treatment naive JIA children may play a significant role in development of autoimmunization and further course of the disease. In conclusion, these results argue for activation of Th17 cells in PB and SF in children with JIA, which may be responsible for the development of autoimmunity and the explosion of local, chronic inflammation in the joints.

Authors declare no conflict of interest.

The study was partially supported by two grants from the Medical University of Lodz: for the Department of Experimental Hematology (No. 503/8-093-01/503-01), and for the Department of Pediatric Cardiology and Rheumatology (No. 502-03/8-000-01/502-64-055), both from Medical University of Lodz, Poland.

\section{References}

1. Lin YT, Wang CT, Gershwin ME, Chiang BL (2011): The pathogenesis of oligoarticular/polyarticular vs systemic juvenile idiopathic arthritis. Autoimmun Rev 10: 482-489.

2. Chabaud M, Durand JM, Buchs N, et al. (1999): Human interleukin-17: a $\mathrm{T}$ cell-derived proinflammatory cytokine produced by the rheumatoid synovium. Arthritis Rheum 42: 963-970.

3. Shen H, Goodall JC, Hill Gaston JS (2009): Frequency and phenotype of peripheral blood Th17 cells in ankylosing spon- 
dylitis and rheumatoid arthritis. Arthritis Rheum 60: 16471656.

4. Nistala K, Wedderburn LR (2008): Th17 and regulatory T cells: rebalancing pro- and anti-inflammatory forces in autoimmune arthritis. Rheumatology 48: 602-606.

5. Chen Z, O'Shea JJ (2008): Th17 cells: a new fate for differentiating helper T cells. Immunol Res 41: 87-102.

6. Niu X, He D, Zhang X, et al. (2010): IL-21 regulates Th17 cells in rheumatoid arthritis. Hum Immunol 71: 334-341.

7. Kleinschek MA, Owyang AM, Joyce-Shaikh B, et al. (2007): IL-25 regulates Th17 function autoimmune inflammation. J Exp Med 204: 161-170.

8. Shen H, Xia L, Lu J, Xiao W (2010): Infliximab reduces the frequency of interleukin 17-producing cells and the amounts of interleukin 17 in patients with rheumatoid arthritis. J Investing Med 58: 905-908.

9. Wehrens EJ, Mijnheer G, Duurland CL, et al. (2011): Functional human regulatory $\mathrm{T}$ cells fail to control autoimmune inflammation due to $\mathrm{PKB} / \mathrm{c}$-akt hyperactivation in effector cells. Blood 118: 3538-3548.

10. Haufe S, Haug M, Schepp C, et al. (2011): Impaired suppression of synovial fluid CD4+CD25- T cells from patients with juvenile idiopathic arthritis by CD4+CD25+ Treg cells. Arthritis Rheum 633: 153-162.

11. Petty RE, Southwood TR, Manners P, et al. (2001): International League of Associations for Rheumatology classification of juvenile idiopathic arthritis: second revision, Edmonton, 2001. J Rheumatol 31: 390-392.

12. Consolaro A, Ruperto N, Bazso A, et al. (2009): Development and validation of a composite disease activity score for juvenile idiopathic arthritis. Arthritis Rheum 61: 658-666.

13. de Kleer IM, Wedderburn LR, Taams LS, et al. (2004): $\mathrm{CD} 4+\mathrm{CD} 25$ bright regulatory $\mathrm{T}$ cells actively regulate inflammation in the joints of patients with remitting from of juvenile idiopathic arthritis. J Immunol 172: 6435-6443.

14. Stelmaszczyk-Emmel A, Jackowska T, Rutkowska-Sak L, et al. (2012): Identification, frequency, activation and function of CD4+CD25 high FoxP3+ regulatory T cells in children with juvenile idiopathic arthritis. Rheumatol Int 32: 11471154 .

15. Han GM, O'Neil-Andersen NJ, Zurier RB, Lawrence DA (2008): CD4+CD25 high T cell numbers are enriched in the peripheral blood of patients with rheumatoid arthritis. Cell Immunol 253: 92-101.

16. Omoyinmi E, Hamaoui R, Pesenacker A, et al. (2012): Th1 and Th17 cell subpopulations are enriched in the peripheral blood of patients with systemic juvenile idiopathic arthritis. Rheumatology 51: 1881-1886.

17. Prado C, de Paz B, Gómez J, et al. (2011): Glucocorticoids enhance Th17/Th1 imbalance and signal transducer and activator of transcription 3 expression in systemic lupus erythematosus patients. Rheumatology 50: 1794-1801.

18. Nistala K, Moncrieffe H, Newton KR, et al. (2008): Interleukin-17-producing T cells are enriched in the joints of children with arthritis, but have a reciprocal relationship to regulatory T cell numbers. Arthritis Rheum 58: 875-887.

19. Ouyang W1, Kolls JK, Zheng Y (2008): The biological functions of $\mathrm{T}$ helper 17 cell effector cytokines in inflammation. Immunity 28: 454-467.

20. Prahalad S, Martins TB, Tebo AE, et al. (2009): Elevated serum levels of soluble CD154 in children with juvenile idiopathic arthritis. Pediatr Rheumatol Online J 28: 1-8.
21. Mahendra A, Misra R, Aggarwal A (2009): Th1 and Th17 Predominance in the Enthesitis-related arthritis form of juvenile idiopathic arthritis. J Rheumatol 36: 1730-1736.

22. Agarwal S, Misra R, Aggarwal A (2008): Interleukin 17 levels are increased in juvenile idiopathic arthritis synovial fluid and induce synovial fibroblasts to produce proinflammatory cytokines and matrix metalloproteinases. J Rheumatol 35: 515-519.

23. de Jager W, Hoppenreijs EP, Wulffraat NM, et al. (2007): Blood and synovial fluid cytokine signatures in patients with juvenile idiopathic arthritis: a cross-sectional study. Ann Rheum Dis 66: 589-598. 